\title{
Towards an Institutional Environment using Norms for Contract Performance
}

\author{
Henrique Lopes Cardoso, Eugénio Oliveira \\ LIACC - NIAD\&R, Faculty of Engineering, University of Porto \\ R. Dr. Roberto Frias, 4200-465 Porto, Portugal \\ hlceipb.pt, eco@fe.up.pt
}

\begin{abstract}
A strong research emphasis is being given towards regulating interoperable multi-agent environments through norms and institutions. We are concerned with environments in which agents form together virtual organizations leading to cooperation agreements that can be enforced. An electronic institution provides a coordination framework facilitating automatic contract establishment and providing an enforceable normative environment. We introduce the notion of contextualized norms within our institutional framework, and develop on a model of institutional reality, taking into account institutional roles and agents' statements, with the aim of providing a contract monitoring service. Our proposal describes how to use norms to formalize cooperation agreements and operational contracts, and how to monitor and detect contract violations.
\end{abstract}

\section{Introduction}

An increasingly important dichotomy in multi-agent systems (MAS) research is autonomy and openness versus regulation. While agent theory puts an emphasis on agents as autonomous self-interested entities interacting in open environments, the application of MAS in real-world scenarios raises an important question: how to ensure an intended cooperative behavior in environments populated with self-interested agents? A possible response to this problem is to regulate the environment, providing incentives for cooperative behavior through normative constraints [2].

In our case, we are concerned with environments in which agents may agree on cooperation efforts, involving specific interactions during a certain time frame. By this way agents may compose a virtual organization (VO), which is regulated by specific and appropriate norms. Here agents usually represent different business entities or enterprises, which come together to address new market opportunities by combining skills, resources, risks and finances no partner alone can fulfill [5].

In open environments, previous performance of potential partners (that is, their reputations) may not be assessed. A VO may comprise agents that have never worked together in the past. This makes it necessary to state, through a formal contract, what an agreement is about, and to provide an environment for enforcing those contracts.

In our view, the Electronic Institution (EI) concept addresses these concerns [10], as it consists of a coordination framework facilitating the establishment of contracts 
and providing a level of trust by offering an enforceable normative environment. This is accomplished through agent-based institutional services, including contract monitoring and enforcement. The EI encompasses a set of norms regulating the environment. However, due to the fact that agents negotiate towards the achievement of agreements formalized in contractual norms, this normative environment is an evolving one. Through appropriate services, the EI monitors and enforces (using sanctions and reputation mechanisms) both institutional norms and those formalizing contracts.

Inside the EI, agents' illocutions are the source towards the formation of institutional reality (inspired in [17]). This reality is composed of both new organizational structures (VOs) and actions performed concerning the compliance to norms. Also, some agents perform specific institutional roles, being certified by the EI as legitimate to produce certain institutional facts. External agents may also announce themselves as performing certain roles. Instead of providing institutional services, these are general roles (such as seller or customer) which may, when performed inside the EI, have a set of attached norms. By assuming those roles, agents become committed to these norms.

In this paper we elaborate on the use of norms within our EI framework (section 2) and on the creation of institutional reality (section 3). Furthermore, we address the specification of contracts (including those devoted to VO settings) using norms regulating behavior (section 4), allowing for contract monitoring and enforcement. Finally we get to some conclusions and identify some related work (section 5).

\section{Contextualized Norms}

Norms play an important role in open artificial agent systems, where they improve coordination and cooperation. As in real-world societies, norms allow us to achieve social order [2] by controlling the environment, making it more stable and predictable.

Norms can be classified according to different criteria. Considering our EI framework, we find it important to classify norms according to their scope [11]. Institutional norms regulate the behavior of every agent inside the EI. By assuming general roles, agents become committed (before the institution) to their associated norms. Institutional norms also include general means of dealing with contract-independent occurrences, such as policies for handling norm violations. They set up the normative ground on which cooperation commitments may be established. Constitutional norms are used to describe the constitution of agent-based virtual organizations, which thereby commit to a certain cooperation agreement. The terms of such an agreement are specified by means of norms regulating the created consortium, which usually exists for a period of time. Finally, operational norms specify contracts by indicating actions to be performed by contractual agents; they may be proposed and signed within the context of a specific VO, or else may comprise a stand-alone deal.

This classification suggests that different types of norms are created at different moments. Thus, institutional norms may be pre-existent, while constitutional norms are created when agents reach cooperation agreements, and operational norms come into existence when executable contracts are signed. However, this needs not be the case. Norms with limited scopes may be predefined for a number of reasons. 
An important concept in contract law theory is the use of "default rules", which facilitate contract formation, allowing contracts to be underspecified by defining default clauses. Therefore, constitutional or even operational norms may be institutionally defined: together with institutional norms, they provide a normative background in which agents can rely to build their contractual commitments.

Furthermore, just as real-world legislations are organized through hierarchies of laws, it is natural to have predefined regulations devoted to particular contexts, such as the VO setting. Agents can rely on these regulations as a ground basis to raise specific virtual organizations.

Finally, norms may be predefined when they regulate predicted coordination situations, as in the case of negotiation protocols. Agents agreeing to coordinate their negotiation efforts according to a certain protocol adhere to the norms implementing it. Differently from "default rules", however, these norms apply as they are to every adhesion to the protocol; they do not make up mere fill-in prescriptions in the absence of explicitly created norms.

According to this setting, it is possible, therefore, to predefine scoped norms that are to be imposed when the activity they regulate is adhered to by agents. This methodology may be applied to negotiation protocols, to standard cooperation commitments between a group of agents, or to norms attached to roles. Although having a limited scope, these norms can be seen as institutional in the sense that they are institutionally predefined.

Independently of the circumstances of their creation, norms define, in some context, what ought to be done in certain circumstances:

$$
\text { [Context] Situation } \rightarrow \text { Prescription }
$$

The Context indicates the scope of the norm. The Situation describes when the norm is in place. The Prescription specifies what should be accomplished.

In all formulae throughout this paper, we use the Prolog notation conventions for variables and relations.

\section{Institutional Reality}

Considering an EI as an environment where social relationships are created and enforced, it is necessary to establish how and when such relationships are in place, and how and when they are fulfilled. If we design a closed EI environment with a welldefined performative structure (as in [14]), agents' actions and their effects are restricted. If, however, we design an open environment where autonomous agents interact, we must relate those interactions with the (emergence of) social structures defining commitments among agents. This represents a much more flexible approach towards the development of a normative framework.

Following Searle's theory on "the construction of social reality" [17], inside the EI we consider brute facts and institutional facts. The latter are obtained from de former, through rules defining "counts-as" relations (constitutive rules). 


\subsection{Brute and Institutional Facts}

Agents' illocutions are stored as brute facts in the form:

$$
\text { illocution(Agent, Content) }
$$

Relevant illocutions are assumed to use a well-defined institutional ontology.

Institutional facts are inferred using constitutive rules. These are fed with agents' illocutions (brute facts), and produce institutional reality. Just as norms have a context in which they apply, we associate institutional facts with the context within which they occur. This is important if we consider facts denoting agent behavior regarding its obligations: these facts may occur within a context (e.g. a contract).

An important issue to consider in contracting scenarios is time: every fact must occur at a given instant. We use the following representation for institutional facts:

$$
\text { [Context] ifact(IFact, Timestamp) }
$$

Just as brute facts, institutional facts are defined in the institutional ontology.

\subsection{The Institutional Reality Engine}

Institutional reality depends on the recording of brute facts, which are then processed by "systems of constitutive rules" [17]. Relevant facts include those related to commitment creation (implying the establishment of contracts) and fulfillment. Therefore, rules regulating how these facts come about are needed.

Institutional Roles. Illocutions' effects may depend on the agents uttering them. We identify a set of institutional roles enacted by agents providing specific services. Some institutional facts may be created only if agents performing certain institutional roles utter appropriate illocutions. Authoritative relations are established in this way between roles and institutional reality: an agent performing a given role is said to be empowered to achieve the effects expressed in its role-related constitutive rules.

Since we are concerned with the application of our model to business scenarios involving transactions, we identify three main institutional roles providing a connection to the real-world. A messenger role provides certified information exchange facilities; a banking role enables acknowledging monetary value transfers; a delivery tracker role certifies product delivery.

Constitutive Rules. Constitutive rules make a connection between what is said and what is taken for granted. Many of these rules will be based on institutional roles and their powers. According to our EI rationale, we identify two main focuses for constitutive rules: (1) the certification of action execution (including contract fulfillment), and (2) the establishment of commitments through contracts.

Certified action execution is important because of trustworthiness issues. Consider a situation in which an agent ought to make a certain payment to another. Although the 
agent may claim to have paid its debt, that does not make it the case. Still, if an independent financial agent, providing a certified institutional service, states that a currency transfer referring to a certain context (e.g. a purchase contract) has taken place, it would be safe to consider that the payment occurred, which is described as below:

illocution(B, currency_transfered(Ctx, Ag1, Amount, Ag2, Time)) ^ibank(B) $\rightarrow[C t x]$ ifact(payment(Ag1, Amount, Ag2), Time)

We can also say that if both agents (the payer and the receiver) acknowledge the payment, it would be safe to conclude the associated institutional fact:

illocution(Ag1, paid(Ctx, Amount, Ag2,_)) ^ illocution(Ag2, collected(Ctx, Amount, Ag1, Time)) $\rightarrow[$ Ctx] ifact(payment(Ag1, Amount, Ag2), Time)

Another exemplifying case where physical actions must be checked concerns the delivery of products. We may trust on a delivery tracking service:

illocution(DT, delivered(Ctx, Ag1, Item, Quantity, Ag2, Time)) ^idelivery_tracker(DT) $\rightarrow[C t x]$ ifact(delivery(Ag1, Item, Quantity, Ag2), Time)

The same methodology can be applied concerning the exchange of messages. If message delivery recognition is a must, an institutional messenger service may be provided. This way, interactions between agents through the exchange of messages can be recorded, as long as such a service intermediates the process.

The messenger agent informs the EI that a given message was delivered. The following constitutive rule applies:

illocution(M, msg_delivered(Ctx, Ag1, Msg, Ag2, Time)) $\wedge$ imessenger(M) $\rightarrow[C t x]$ ifact $(m s g(A g 1, M s g, A g 2)$, Time $)$

In principle, any information exchange could be treated in a similar way. This opens up the possibility to verify business-related activities such as order placement, invoice issuing, or shipment notices. It also enables the verification of negotiation protocols requiring the exchange of proposals.

As for contract establishment, we must define relations between institutional facts (created from agents' illocutions) and commitment formation. These constitutive rules define how new institutionally enforceable norms can be created, describing contractual relationships between agents.

Contractual relationships may rise from an appropriate exchange of messages. For instance, a contract may be recognized if an agent accepts the terms and conditions of a standing proposal (that is, when agents reach an agreement):

ifact(acceptation(Ag1, Ag2), TA) ^ ifact(proposal(Ag2, Ag1, Proposal, Timeout), TP) ^ $T P<T A<T P+$ Timeout $\rightarrow$ register_new_contract $(A g 1$, Ag2, Proposal, $T A)$ 
where register_new_contract would be an institutional procedure registering the contract between the involved agents. Several researchers address the issue of commitment creation from interaction protocols. A survey may be found in [12].

If, however, negotiation protocols must be enforced, or if negotiation must be mediated (besides mere message forwarding), negotiation mediation services may be provided by an institutional agent. This applies to negotiation protocols specifically devoted to formalizing VO cooperation agreements, as we have proposed before [13].

\section{Specifying and Monitoring Contracts}

Behavior norms prescribe the expected behavior of agents. We attribute the responsibility of monitoring and enforcing norms to the EI, which by this means establishes a trust-enabled normative environment.

A norm-aware environment can operate either preventively (making unwanted behavior impossible) or responsively (detecting violations and reacting accordingly) [18]. Taking into account the autonomous nature of agents, we rely essential on the latter practice. Norms specify states of affairs that must be brought about by an agent before a certain deadline. Therefore, we consider obligations as the means to express the prescription of behavior norms. Obligations have the following structure:

[Context] obligation(Bearer, InstitutionalFact, Deadline)

Instead of dictating the exact action an agent must perform, we prescribe the institutional fact that it must bring about. This fits with our model of institutional reality, where we specify through constitutive rules how an institutional fact may be accrued. It also enables an agent to delegate tasks conducting to the accomplishment of such state of affairs, while still being responsible for the (un)fulfillment of the obligation.

Situations in which norms apply include the achievement of institutional facts, and the fulfillment or violation of obligations. Norms prescribing behavior in case of violations are sanctioning norms: they are meant to discourage non-compliance. These norms may be defined either as institutional or as contract-specific.

\subsection{Fulfillment and Violation of Obligations}

Contextualized institutional facts are used to verify the fulfillment of obligations. For this, we define an obligation fulfillment rule:

[Context] ifact(IFact, T) ^obligation(Bearer, IFact, Deadline) $\wedge$ T<Deadline

$\rightarrow$ fulfilled(Bearer, IFact, $T$ )

This rule indicates that if an institutional fact prescribed by an obligation is achieved before its deadline, then that obligation is fulfilled. Literals within the rule are dependent on its context. However, this rule is institutional, as it applies to all contractual relations; it thus has un-instantiated Context. 
This rule is fundamental for enabling the chaining of obligations within a contractual relationship. It establishes a connection between the institutional facts that are added and the pending obligations.

Sanctioning norms are activated using a violation detection rule, which fires when deadlines have elapsed. Time events are generated as institutional facts referring to the time when obligations are due.

[Context] ifact(time, Deadline) $\wedge$

obligation(Bearer, IFact, Deadline) ^ not(fulfilled(Bearer, IFact,_))

$\rightarrow$ violated(Bearer, IFact, Deadline)

This violation detection rule states that in any context, if a deadline referring to an obligation was reached, and such obligation was not fulfilled, then a violation occurred. The resulting fact may be used to activate sanctioning norms and to update the agent's reputation.

This approach allows us to distinguish violation detection from sanction imposition mechanisms. While the detection of violations is a general and institutionally defined concept, the prescription of sanctions may be contract-specific.

\subsection{Virtual Organization Cooperation Agreements}

A cooperation agreement aggregates the VO's constitutional information, including the cooperation effort agents are committed to, and their general business process flow. Considering situations where the intended cooperation consists of the exchange of resources, the following templates are used to specify this information:

[] ifact(cooperation_agreement(IdCA, Participants, Resources), CATime)

[cooperation_agreement:IdCA]

coop_effort(Participant, Resource, MinQuantity, MaxQuantity, Frequency, UnitPrice)

[cooperation_agreement:IdCA] business_process(From, Resource, To)

Cooperation efforts indicate, for each participating agent, quantity ranges for the supply of resources, within a given frequency, together with agreed prices. Business process entries indicate the resources that are supposed to flow between participants. Their effective transfer, however, is dependent on appropriate requests.

The central norm in respect to contractual promises indicates that each agent is committed to its cooperation effort. This translates to an obligation prescription:

[cooperation_agreement:IdCA]

ifact(request(Requester, Resource, Quantity, Answerer), TR)

business_process(Answerer, Resource, Requester) $\wedge$

coop_effort(Answerer, Resource, MinQt, MaxQt, Freq, _) $\wedge$

calculate_performed_effort(Answerer, Resource, Freq, TR, PE) $\wedge P E+Q u a n t i t y<=$ Max $Q t$

$\rightarrow$ obligation(Answerer, acknowledge(Answerer, Resource, Quantity, Requester), TR+10) 
This norm is institutionally defined: it applies to all cooperation agreements created inside the institution. Its context remains unbound until it is in use. Briefly, it states that if a predicted request (considering the stated business process and cooperation effort) is made in the context of a cooperation agreement, then the envisaged agent is obliged to accept it. An institutional procedure (calculate_performed_effort) is invoked for calculating the effort already performed by the agent. If it does not exceed its promised efforts, the obligation comes into effect.

\subsection{Operational Contracts}

Operational contracts are established in the context of a cooperation agreement. Institutional facts register their creation:

[cooperation_agreement:IdCA]

ifact(operational_contract(IdOC, Requester, Answerer, Resource, Quantity), OCTime)

Considering parties' cooperation commitments, operational contracts come into existence through a constitutive rule of the form:

[cooperation_agreement:IdCA]

fulfilled(Answerer, acknowledge(Answerer, Resource, Quantity, Requester), TA)

$\rightarrow$ register_new_operational_contract(IdCA, Requester, Answerer, Resource, Quantity, TA)

This rule applies to every cooperation agreement, and states that when an agent fulfils its obligation to acknowledge a given request, a new operational contract comes into existence.

The cooperation agreement may also specify how operational contracts are managed, that is, what obligation chains implement such activity. This facilitates their creation, since their norms may be pre-specified. One possibility is to define norms applicable to all operational contracts within a cooperation agreement. For instance, if delivery and payment should occur:

[cooperation_agreement:IdCA, operational_contract:IdOC]

obligation(Answerer, delivery(_, Resource, Quantity, Requester), OCTime+10)

[cooperation_agreement:IdCA, operational_contract:IdOC]

fulfilled(Answerer, delivery(_,Resource, Quantity, Requester), TD) $\wedge$

coop_effort(Answerer, Resource, _, _, , UnitPrice)

$\rightarrow$ obligation(Requester, payment(_, UnitPrice*Quantity, Answerer), TD+30)

where $I d O C$ remains unbound, as these norms apply to all operational contracts which will be created in the future within agreement IdCA.

It would also be possible to define an institutional default policy, applicable to all operational contracts of all cooperation agreements. 


\section{Conclusions and Related Work}

The regulation of multi-agent systems in environments with no central design (and thus with no cooperative assumption) is gaining much attention in the research community. Normative multi-agent systems address this issue by introducing incentives to cooperation (or discouraging deviation). After initial research on norms as constraints on behavior, it is now accepted that autonomous agents are able to deliberate whether to comply with norms [3].

Searle's work on speech acts [16] and institutional reality [17] has inspired several researchers within the MAS field (e.g. [1], [4], and [7]). In our case, we used this inspiration as a means to certify the occurrence of real-world actions, essential to contract monitoring purposes. Brute facts consist of agents' illocutions, which according to empowering relations are used to produce institutional reality.

Within the framework of our proposed electronic institution [10] providing services for the achievement of contractually specified agreements (including VO scenarios), we described the use of contextualized norms and the specification and monitoring of contracts. Norms are typically related with the deontic notions of obligation, permission and prohibition, and have been used to formalize contracts (e.g. [9] and [15]). In our case, we essentially rely on directed obligations. In the case of VO contracts, permissions are seen as rights for requesting a partner's contribution. Prohibitions may be applied as a consequence of violation detection. A formal underpinning of a logic for contract representation is given in [6], including the notion of conditional obligation with deadline (equivalent to our norm specification).

The concept of electronic institutions is gaining importance inside MAS research. Previous approaches towards regulating agent behavior through EIs include [14]. However, this model formally defines an institution using a rigid structure that implements a predefined protocol. By restricting the actions agents are allowed to perform, it does not cope well with a central property of agency: autonomy. In our approach, we avoid imposing hard constraints on behavior. By enforcing norms, we do conduct and supervise the behavior of rational agents.

Top-down normative frameworks are appropriate in situations where norms can be centrally designed, although regulating a distributed environment with autonomous self-interested agents. However, it is not amenable to contract handling: agents make agreements that are to be monitored by a trusted third-party (the EI), thus the normative structure is modified by the corresponding contracts. We aim at providing such an evolving normative framework, taking into account the creation of institutional reality.

Norms lend themselves to a rule-based implementation. We are implementing our agent-based EI framework in JADE and using the JESS shell [8] for norm representation, monitoring and enforcement. JESS incorporates features enabling also the use of default reasoning, and consists of a forward-chaining production system that fits the firing of norms and rules based on events (institutional facts in our case). It also includes the possibility to define modules for organizing sets of rules, matching our norm contexts. 


\section{References}

1. Boella, G., \& van der Torre, L. (2004). Regulative and Constitutive Norms in Normative Multiagent Systems. In Proc. 9th Int. Conf. on the Principles of Knowledge Representation and Reasoning (KR'04), Whistler, Canada.

2. Castelfranchi C. (2000). Engineering Social Order. In A. Omicini, R. Tolksdorf, \& F. Zambonelli (eds.), Engineering Societies in the Agents World, Springer, pp.1-18.

3. Castelfranchi, C., Dignum, F., Jonker, C., \& Treur, J. (2000). Deliberative Normative Agents: Principles and Architectures. In N. Jennings \& Y. Lesperance (eds.), Intelligent Agents VI: Agent Theories, Architectures, and Languages, Springer, pp.364-378.

4. Colombetti, M., \& Verdicchio, M. (2002). An analysis of agent speech acts as institutional actions. In Castelfranchi \& Johnson (eds.), Proc. 1st Int. Joint Conf. on Autonomous Agents and Multiagent Systems, ACM Press, pp.1157-1164.

5. Dignum, V., \& Dignum, F. (2002). Towards an Agent-based Infrastructure to Support Virtual Organizations. In L.M. Camarinha-Matos (ed.), Collaborative Business Ecosystems and Virtual Enterprises, Kluwer, pp.363-370.

6. Dignum, V., Meyer, J.-J., Dignum, F., \& Weigand, H. (2003). Formal Specification of Interaction in Agent Societies. In M. Hinchey, J. Rash, W. Truszkowski, C. Rouff \& D. GordonSpears (eds.) Formal Approaches to Agent-Based Systems, Springer, pp.37-52.

7. Fornara, N., Viganò, F., \& Colombetti, M. (2005). Agent Communication and Institutional Reality. In R. M. van Eijk, M.-P. Huget \& F. Dignum (eds.), Agent Communication, Springer, pp.1-17.

8. JESS, the Rule Engine for the Java Platform (http://herzberg.ca.sandia.gov/jess/).

9. Kollingbaum, M.J., \& Norman, T.J. (2002). Supervised Interaction - Creating a Web of Trust for Contracting Agents in Electronic Environments. In Castelfranchi \& Johnson (eds.), Proc. 1st Int. Joint Conf. on Autonomous Agents and Multiagent Systems, ACM Press, pp.272-279.

10. Lopes Cardoso, H., Malucelli, A., Rocha, A.P., \& Oliveira, E. (2005). Institutional Services for Dynamic Virtual Organizations. In Proc. 6th IFIP Working Conference on Virtual Enterprises (PRO-VE'05), Valencia, Spain, 26-28 September 2005.

11. Lopes Cardoso, H., \& Oliveira, E. (2005). Virtual Enterprise Normative Framework within Electronic Institutions. In M.-P. Gleizes, A. Omicini \& F. Zambonelli (eds.), Engineering Societies in the Agents World V, Springer, pp.14-32.

12. Maudet, N., \& Chaib-draa, B. (2002). Commitment-based and dialogue-game based protocols - new trends in agent communication languages, Knowledge Engineering 17(2), pp.157-179.

13. Oliveira, E., \& Rocha, A.P. (2000). Agents Advanced Features for Negotiation in Electronic Commerce and Virtual Organisations Formation Process. In F. Dignum \& C. Sierra (eds.), Agent Mediated Electronic Commerce: The European AgentLink Perspective, Springer, pp. 78-97.

14. Rodríguez-Aguilar, J.A. (2001). On the design and construction of Agent-mediated Electronic Institutions, Ph.D. Thesis, Universitat Autònoma de Barcelona.

15. Sallé, M. (2002). Electronic Contract Framework for Contractual Agents. In R. Cohen \& B. Spencer (eds.), Advances in Artificial Intelligence: 15th Conference of the Canadian Society for Computational Studies of Intelligence, Springer, pp.349-353.

16. Searle, J.R. (1969). Speech Acts: an Essay in the Philosophy of Language. Cambridge, England: Cambridge University Press.

17. Searle, J.R. (1995). The Construction of Social Reality, Free Press: New York.

18. Vázquez-Salceda, J., Aldewereld, H., \& Dignum, F. (2004). Implementing norms in multiagent systems. In G. Lindemann, J. Denzinger, I. J. Timm \& R. Unland (eds.), Multiagent System Technologies, Springer, pp.313-327. 